\title{
HOMEOPATIA E SUPLEMENTO ALIMENTAR NO CRESCIMENTO DE FILHOTES DE PERIQUITO AUSTRALIANO (MELOPSITTACUS UNDULATUS)
}

\author{
Jéssica da Silva Martins ${ }^{1}$, Vanair Carlos Paz ${ }^{2}$, Rodrigo Diana Navarro ${ }^{1}$
}

\begin{abstract}
RESUMO - Objetivou-se avaliar o crescimento de filhotes de Periquitos Australianos (Melopsittacus undulatus) tratados com homeopatias Calcarea carbonica $(12 \mathrm{CH})$ e Carbo vegetalis $(12 \mathrm{CH})$ e o suplemento alimentar Organew ${ }^{\circledR}$. O experimento foi feito a partir de um delineamento inteiramente casualizado com 3 diferentes tratamentos e 4 repetições, sendo o tratamento 1: suplemento alimentar, tratamento 2: homeopatia e o tratamento 3: controle. Os resultados da análise de peso mostraram que os filhotes que receberam o suplemento alimentar tiveram um maior ganho de peso $(33,8 \mathrm{~g})$, seguidos dos animais que receberam a homeopatia $(33,6 \mathrm{~g})$ e por último o grupo controle $(30,2 \mathrm{~g})$. A média de ganho de peso diário dos filhotes do grupo com suplemento alimentar foi de 1,69 g, do grupo com homeopatia, 1,68 g, e o grupo controle, 1,51 g.
\end{abstract}

Palavras chave: filhotes, homeopatia, Melopsittacus Undulatus, suplemento alimentar.

\section{HOMEOPATHY AND FOOD SUPPLEMENT IN THE GROWTH OF AUSTRALIAN PERIQUITO CUBS (MELOPSITTACUS UNDULATUS)}

\begin{abstract}
The objective of this work was to analyze and evaluate the growth of Australian Parakeets (Melopsittacus undulatus) treated with Calcarea carbonica $(12 \mathrm{CH})$ and Carbo vegetalis (12 CH) homeopathies and the Organew ${ }^{\circledR}$ food supplement. The experiment was made from a completely randomized design with 3 different treatments and 4 replicates, being the treatment 1: food supplement, treatment 2: homeopathy and the treatment 3: control. The results of the weight analysis show that the babies receiving the food supplement have a higher gain of weight (33.8g), followed by the animals that received a homeopathy $(33.6 \mathrm{~g})$ and finally the control group (30.2g). An average of daily weight gain of the group of babies with food supplement was $1.69 \mathrm{~g}$, group with homeopathy was $1.68 \mathrm{~g}$ and control group was $1.51 \mathrm{~g}$.
\end{abstract}

Keywords: feeding supplement, homeopathy, Melopsittacus undulates, puppies.

\section{INTRODUÇÃO}

A ordem Psittaciformes é constituída por três famílias: 1) Loridae, composta pelos lóris; 2) Cacatuidae, pelas cacatuas e calopsitas e 3) Psittacidae, pelas araras, papagaios e periquitos. Existem 375 espécies de psittaciformes reconhecidas no mundo, sendo que dessas, 85 ocorrem no Brasil. (Grespan e Raso, 2014)

O Periquito Australiano (Melopsittacus undulatus), descrito pela primeira vez por Shaw em 1850, é uma ave pertencente a ordem Psittaciforme e família Psittacidae. Nativo da Austrália é encontrado principalmente no interior do país (Forshaw, 1973). Devido a sua criação como animal de estimação, hoje pode ser encontrado em lares em todos os continentes.

A criação do Melopsittacus como animal doméstico foi listada em 7 de julho de 1998, quando o IBAMA publicou a portaria $\mathrm{n}^{0} 93 / 1998$, regulamentadora da importação e exportação da fauna silvestre e postou uma lista de animais exóticos autorizados a serem criados como domésticos no Brasil. Desde então, essas pequenas aves são vendidas em praticamente todas as casas agropecuárias e se tornaram muito populares, entre os brasileiros, devido a sua docilidade e sociabilidade.

Avanços na medicina permitiram o surgimento de novas áreas e especialidades. Como alternativa aos tratamentos

\footnotetext{
1 Faculdade de Agronomia e Medicina Veterinária, Universidade de Brasília, Brasília, DF, Brazil. E-mail: navarrounb@gmail.com.

2 Criadouro Vanair aves, Planaltina - DF.
} 
da medicina convencional, que deixam resíduos químicos e tóxicos no corpo e no ambiente, surgiu a Homeopatia.

Hipócrates, pai da medicina, concluiu que "similia similibus curentur" (sejam os semelhantes curados pelos semelhantes), que foi a base da Homeopatia. (Neto, 2006). $\mathrm{Na}$ homeopatia, o indivíduo é visto como uma unidade formada de corpo, mente e emoção, logo o organismo não adoece em algumas partes, adoece como um todo (Hahnemann, 1996).

Segundo Moreno (2008), a Calcarea carbonica favorece a absorção de alimentos e eliminação, favorecendo o sistema imunológico. Permite viver com menos vírus, fungos, bactérias e vermes. Fortalece ossatura, evita fraturas e a disponibilidade de cálcio fica aumentada de forma a equilibrar o animal.

Ainda segundo Moreno (2008), o Carbo vegetabilis é preventivo de doenças, limpa o sistema circulatório e linfático e age rápido em casos de emergência em que o animal está a beira da morte, fortalecendo e dando disposição.

Objetivou-se analisar e comparar o crescimento de filhotes de Periquitos Australianos (Melopsittacus undulatus) tratados com as homeopatias Calcarea carbonica $(12 \mathrm{CH})$ e Carbo vegetalis $(12 \mathrm{CH})$ e o suplemento alimentar Organew ${ }^{\circledR}$.

\section{MATERIAL E MÉTODOS}

\section{Local do estudo}

Este trabalho foi aprovado pelo Comitê de Ética no Uso Animal (CEUA), protocolo n. ${ }^{\circ}$ 96/2017. O trabalho foi desenvolvido no Criadouro Vanair Aves, localizado em Planaltina/DF (latitude 15'38'25' S e longitude $47^{\circ} 41^{\prime} 7^{\prime}$ 'W). O estudo foi realizado entre os meses de agosto e outubro de 2017. A temperatura média durante o período de coleta dos dados foi de $27^{\circ} \mathrm{C} \pm 4^{\circ} \mathrm{C}$.

$\mathrm{O}$ experimento foi conduzido num Delineamento Inteiramente Casualizado com três diferentes tratamentos e quatro repetições, sendo 1) Probiótico Organew, 2) homeopatia e 3 ) controle.

Os animais foram mantidos em gaiolas $(0,45 \mathrm{~m} \mathrm{x}$ $0,24 \mathrm{~m} \times 0,33 \mathrm{~m}$ ), que foram mantidas dentro de um galpão de alvenaria, com uma das paredes de tela metálica com cortinas de lona plástica e com telhado de telhas de barro e algumas telhas de acrílico transparente para entrada de luz. Os locais dentro da gaiola estão detalhados na Tabela 1.

Foram utilizadas 12 gaiolas, equipadas com um comedouro $(12,5 \mathrm{~cm} \times 7,5 \mathrm{~cm} \times 4 \mathrm{~cm})$, cada uma com um casal de Periquitos Australianos e seis filhotes no ninho $(0,18 \mathrm{~m} \times 0,18 \mathrm{~m} \times 0,20 \mathrm{~m})$, totalizando 96 aves.

A farinhada foi composta de flocos de arroz, flocos de milho e ovo em pó (Tabela 2). O suplemento alimentar utilizado no experimento foi o Organew ${ }^{\circledR}$ (Tabela 3), com adição de ginseng. As homeopatias utilizadas foram a Calcarea carbonica (CH 12) e o Carbo vegetalis (CH 12). Adicionou-se 1 gota de cada nos bebedouros $(100 \mathrm{~mL}) \mathrm{com}$ água trocada todos os dias pela manhã.

Tabela 1 - Descrição dos locais dentro da gaiola

\begin{tabular}{lll}
\hline Sigla & \multicolumn{1}{c}{ Local } & Descrição \\
\hline GN & $\begin{array}{l}\text { Grade lateral } \\
\text { do ninho }\end{array}$ & $\begin{array}{l}\text { Estrutura de arame de aço } 3,5 \mathrm{~mm} \text { de } \\
\text { diâmetro, de cor branca }(0,5 \times 0,5 \mathrm{~m}) ; \\
\text { espaçamento entre as barras de 1,6cm; } \\
\text { abertura para encaixe do ninho. }\end{array}$ \\
\hline PS & $\begin{array}{l}\text { Poleiro } \\
\text { superior }\end{array}$ & $\begin{array}{l}\text { Estrutura de madeira com 0,5m de } \\
\text { comprimento e 1,6cm de diâmetro; acoplado } \\
\text { transversalmente à 40cm do chão da gaiola. }\end{array}$ \\
\hline PI & $\begin{array}{l}\text { Poleiro } \\
\text { inferior }\end{array}$ & $\begin{array}{l}\text { Estrutura de madeira com 0,5m de } \\
\text { comprimento e 1,6cm de diâmetro; } \\
\text { acoplado transversalmente à 20cm do chão } \\
\text { da gaiola. }\end{array}$ \\
\hline N & Ninho & $\begin{array}{l}\text { Estrutura de madeira tipo caixa (18cm de } \\
\text { comprimento, 18cm de largura e 20cm de } \\
\text { altura) com porta lateral e superior do tipo } \\
\text { dobradiça e abertura voltada para dentro da } \\
\text { gaiola com 6cm de diâmetro; Cobertura do } \\
\text { ninho com maravalha. }\end{array}$ \\
\hline CS & $\begin{array}{l}\text { Comedouro } \\
\text { de sementes }\end{array}$ & $\begin{array}{l}\text { Comedouro de plástico de cor branca } \\
\text { (12,5cm de comprimento, 7,5 cm de largura } \\
\text { e 4 cm de altura). }\end{array}$ \\
\hline CF & $\begin{array}{l}\text { Comedouro } \\
\text { de farinhada }\end{array}$ & $\begin{array}{l}\text { Comedouro de plástico de cor preta }(5 \mathrm{~cm} \\
\text { de comprimento, 4cm de largura e 2,5cm de } \\
\text { altura) de encaixe na grade da gaiola. }\end{array}$ \\
\hline B & Bebedouro & $\begin{array}{l}\text { Bebedouro de plástico de cor leitosa de } \\
\text { encaixe na grade da gaiola. 100 mL. }\end{array}$ \\
\hline & &
\end{tabular}

Tabela 2 - Análise bromatológica dos alimentos utilizados

\begin{tabular}{lcccc}
\hline Amostra & $\begin{array}{c}\text { Matéria seca } \\
105^{\circ} \mathrm{C} \text { MS } \\
(\%)\end{array}$ & $\begin{array}{c}\text { Matéria } \\
\text { Mineral MM } \\
(\%)\end{array}$ & $\begin{array}{c}\text { Extrato } \\
\text { Etéreo EE } \\
(\%)\end{array}$ & $\begin{array}{c}\text { Proteína } \\
\text { Bruta (\%) }\end{array}$ \\
\hline Arroz & $88,84 \pm 0,001$ & $4,71 \pm 0,002$ & $0,92 \pm 0,000$ & $7,65 \pm 0,352$ \\
Painço & $89,07 \pm 0,000$ & $1,97 \pm 0,000$ & $0,52 \pm 0,000$ & $14,53 \pm 0,031$ \\
Aveia & $88,65 \pm 0,001$ & $1,69 \pm 0,001$ & $1,78 \pm 0,000$ & $16,80 \pm 0,000$ \\
Girassol & $91,66 \pm 0,000$ & $2,23 \pm 0,002$ & $3,71 \pm 0,000$ & $15,05 \pm 0,814$ \\
Farinhada & $89,88 \pm 0,002$ & $4,15 \pm 0,004$ & $1,07 \pm 0,000$ & $14,07 \pm 0,701$ \\
\hline
\end{tabular}

Laboratório de Análise de Alimento da UPIS, Campus II, Planaltina, DF (conforme os procedimentos da AOAC, 1995). 
Tabela 3 - Níveis de garantia do suplemento alimentar Organew ${ }^{\circledR}$

\begin{tabular}{lc}
\hline Proteína bruta (Mín.) & $178,6 \mathrm{~g}$ \\
Matéria fibrosa (Máx.) & $500 \mathrm{mg}$ \\
Extrato etéreo (Mín.) & $28,3 \mathrm{~g}$ \\
Umidade & $73,9 \mathrm{~g}$ \\
Vitamina B1 & $5,4 \mathrm{mg}$ \\
Vitamina B2 & $19 \mathrm{mg}$ \\
Vitamina B6 & $11,2 \mathrm{mg}$ \\
Vitamina B12 & $24 \mathrm{mcg}$ \\
Biotina & $2,8 \mathrm{mg}$ \\
Ácido fólico & $10 \mathrm{mg}$ \\
Cistina & $2,461 \mathrm{mg}$ \\
Glicina & $8,481 \mathrm{mg}$ \\
Colina & $750 \mathrm{mg}$ \\
Isoleucina & $8,740 \mathrm{mg}$ \\
Lisina & $16,68 \mathrm{~g}$ \\
Leucina & $13,3 \mathrm{~g}$ \\
Serina & $9,803 \mathrm{mg}$ \\
Metionina & $6,649 \mathrm{mg}$ \\
Tirosina & $4,570 \mathrm{mg}$ \\
Ácido glutâmico & $20,36 \mathrm{~g}$ \\
Triptofano & $1,8 \mathrm{~g}$ \\
Ácido aspártico & $18,11 \mathrm{~g}$ \\
FOS & $2 \mathrm{~g}$ \\
MOS & $1 \mathrm{~g}$ \\
Saccharomyces cerevisiae & $9 \times 109 \mathrm{UFC}$ \\
Cálcio (Máx.) & $0,18 \%$ \\
Fósforo (Mín.) & $0,02 \%$ \\
Veículo q.s.p & $1.000 \mathrm{~g}$ \\
\hline Font: (htp:/Ww.rot \\
\hline
\end{tabular}

Fonte: (http://www.vetnil.com.br/produtos/organew-2/).

Quatro gaiolas foram separadas para cada tratamento. Toda as aves receberam diariamente $56 \mathrm{~g}$ de mistura de sementes sendo elas painço $(60 \%)$, arroz $(10 \%)$, aveia $(5 \%)$, girassol $(10 \%)$, alpiste $(10 \%)$ e Niger $(5 \%)$. As aves do tratamento 1 receberam $19 \mathrm{~g}$ de farinhada contendo o suplemento. As aves do tratamento 2 receberam a homeopatia diluída em $100 \mathrm{~mL}$ de água e as aves do tratamento 3 receberam $19 \mathrm{~g}$ de farinhada sem adição do suplemento. Diariamente, os restos de alimentos, principalmente cascas das sementes, dentro dos comedouros foram pesados individualmente para coleta de dados de consumo diário.

\section{Coleta dos dados}

Os dados comportamentais foram coletados no período de 60 dias perfazendo uma média de 30 horas de amostragem e um total de 1800 registros. O método de anotação dos dados foi o animal focal com registro instantâneo, com intervalos de 1 minuto durante 2 horas por dia (Altemann, 1974). Os animais foram observados entre duas e sete vezes por semana, sendo as observações distribuídas no período da manhã (entre 11h e 14h).

Os filhotes foram pesados ao início do estudo, depois com 10 dias e ao final do experimento com 20 dias. Foram realizados dois exames parasitológicos nas aves, ao início e ao fim do tratamento. O protocolo foi o Método de Faust, utilizado para o diagnóstico de Giardia sp., ovos leves, cistos e oocistos (Faust et al., 1938).

Seguindo o método de Faust, utilizou-se cerca de 1 grama de fezes frescas e colocou-se em um béquer com o auxilio de uma palheta de madeira, homogeneizouse com cerca de $5 \mathrm{~mL}$ de água da bica e após a ausência ou quantidade desprezível de resíduos sólidos verteu-se a solução para um tubo de ensaio específico para centrifugar. Centrifugou-se por 2 minutos a $2500 \mathrm{rpm}$, descartou-se o sobrenadante, essa etapa foi repetida até ser observada pouca coloração no sobrenadante. Desprezou-se então o sobrenadante e adicionou-se $5 \mathrm{~mL}$ de uma solução de sulfato de zinco, com o auxílio de uma pipeta Pasteur, e centrifugou-se novamente por 2 minutos a $2500 \mathrm{rpm}$. Retirou-se três pequenas alíquotas da superfície do material e colocou-se na lâmina, cobriu-se então a região com a lamínula e observou-se em um microscópio.

O escore corporal das aves foi classificado como bom $(3,5)$, em uma escala de $0-5$, de acordo com avaliação visual de deposição de musculatura no peito.

\section{Análise dos dados}

Após a coleta de dados, o processamento estatístico de consumo diário, dados comportamentais e dados biométricos foram feitos através da análise de variância ANOVA One-way pelo programa PAST® (Hammer \& Haper, 1999).

\section{RESULTADOS}

Não foi observada presença de ovos, cistos ou larvas de giardia $s p$. nas aves nos diferentes tratamentos (Tabela 4). 
Tabela 4 - Resultado dos exames parasitológicos

\begin{tabular}{lcc}
\hline Tratamento & Coleta inicial & Coleta final \\
\hline Suplemento alimentar & Negativo & Negativo \\
Homeopatia & Negativo & Negativo \\
Controle & Negativo & Negativo \\
\hline
\end{tabular}

Os resultados da análise de peso mostraram que os filhotes que receberam suplemento alimentar tiveram um maior ganho de peso $(34,17 \mathrm{~g})$, seguidos dos animais que receberam a homeopatia $(33,61 \mathrm{~g})$, e por último o grupo controle $(32,45 \mathrm{~g})$ (Tabela 5 e 6$)$. A média de ganho de peso diário dos filhotes do grupo com suplemento alimentar foi de $1,7 \mathrm{~g}$, do grupo com homeopatia foi de $1,68 \mathrm{~g}$ e o grupo controle 1,62 g (Tabela 7). As variações do ganho de peso estão na Figura 1.

O peso dos pais também foi avaliado no início e ao final. (Tabela 8), juntamente com o consumo de sementes e farinhada, e ainda foi calculado o ganho de peso diário dos filhotes. Médias na mesma coluna seguidas de letras distintas diferem ao nível de $5 \%$ de significância pelo teste ANOVA one-way.

Tabela 5 - Peso dos filhotes de Melopsittacus undulatus no período de experimento, médias e desvio padrão

\begin{tabular}{lccc}
\hline Tratamento & $1^{\circ}$ dia & 10 dias & 20 dias \\
\hline $\begin{array}{l}\text { Suplemento } \\
\text { alimentar }\end{array}$ & $3,16 \pm 0,9 \mathrm{a}$ & $21,33 \pm 2.51 \mathrm{a}$ & $37,33 \pm 0,5 \mathrm{a}$ \\
Homeopatia & $2,57 \pm 0,3 \mathrm{a}$ & $20,09 \pm 1,95 \mathrm{~b}$ & $36,18 \pm 3,0 \mathrm{~b}$ \\
Controle & $2,15 \pm 0,2 \mathrm{~b}$ & $19,11 \pm 1,01 \mathrm{c}$ & $34,6 \pm 3,3 \mathrm{c}$ \\
\hline
\end{tabular}

Médias na mesma coluna seguidas de letras distintas diferem ao nível de 5\% de significância pelo teste ANOVA one-way.
Tabela 6 - Ganho de peso médio dos filhotes de Melopsittacus undulatus, seguidos pelo desvio padrão

\begin{tabular}{lc}
\hline Tratamento & Ganho de peso $(\mathrm{g})$ \\
\hline Suplemento alimentar & $34,17 \pm 0,70 \mathrm{a}$ \\
Homeopatia & $33,61 \pm 2,49 \mathrm{~b}$ \\
Controle & $32,45 \pm 3,60 \mathrm{c}$ \\
\hline
\end{tabular}

Médias na mesma coluna seguidas de letras distintas diferem ao nível de 5\% de significância pelo teste ANOVA one-way.

Tabela 7 - Média de ganho de peso diário dos filhotes nos diferentes tratamentos

\begin{tabular}{lc}
\hline Tratamentos & Ganho de peso diário $(\mathrm{g})$ \\
\hline Suplemento alimentar & 1,7 \\
Homeopatia & 1,68 \\
Controle & 1,62 \\
\hline
\end{tabular}

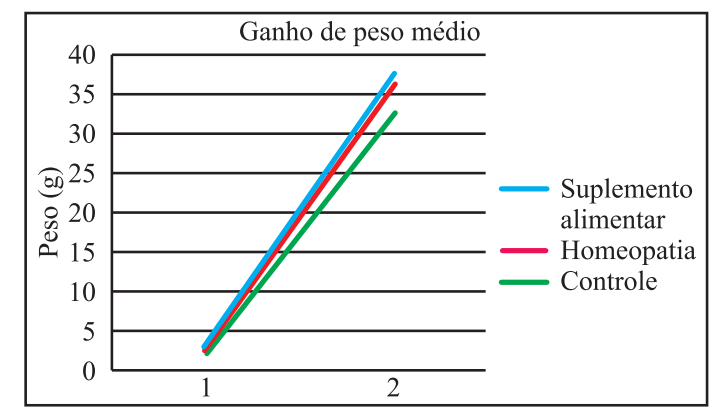

Figura 1 - Ganho de peso médio dos filhotes de Melopsittacus undulatus.

Tabela 8 - Media de peso (em gramas) dos reprodutores de Melopsittacus undulatus, seguidos pelo desvio padrão

\begin{tabular}{|c|c|c|c|c|}
\hline Tratamento & Peso inicial fêmeas & Peso final fêmeas & Peso inicial machos & Peso final machos \\
\hline Suplemento alimentar & $43,75 \pm 1,52 \mathrm{a}$ & $39,25 \pm 3,5 \mathrm{a}$ & $39,75 \pm 6,02 \mathrm{a}$ & $37,5 \pm 5,80 \mathrm{a}$ \\
\hline Homeopatia & $42,5 \pm 3,51 \mathrm{a}$ & $37,5 \pm 4,04 a$ & $40 \pm 2,51 \mathrm{a}$ & $38 \pm 1,63 a$ \\
\hline Controle & $41,25 \pm 1,73 a$ & $35,5 \pm 2,64 \mathrm{a}$ & $39,75 \pm 5,29 a$ & $37,5 \pm 1,89 \mathrm{a}$ \\
\hline
\end{tabular}

Médias na mesma coluna seguidas de letras distintas diferem ao nível de $5 \%$ de significância pelo teste ANOVA one-way.

O consumo de farinhada foi semelhante nos três tratamentos, a análise de variância não identificou diferença significativa $(p>0,05)$ entre as médias de consumo de farinhada, porém o consumo de sementes foi maior pelo grupo que recebeu o suplemento alimentar, seguido pelo grupo controle. $\mathrm{O}$ grupo que recebeu homeopatia foi o que teve menor consumo de sementes (Tabela 9). 
Tabela 9 - Média de consumo dos reprodutores Melopsittacus undulatus de farinhada e sementes nos três tratamentos, seguidos pelo desvio padrão

$\begin{array}{lccc}\text { Alimento } & \text { Suplemento alimentar }(\mathrm{g}) & \text { Homeopatia }(\mathrm{g}) & \text { Controle }(\mathrm{g}) \\ \text { Sementes } & 38,24 \pm 13,16 \mathrm{a} & 30,40 \pm 9,30 \mathrm{~b} & 34,37 \pm 7,86 \mathrm{a} \\ \text { Farinhada } & 10,14 \pm 4,61 \mathrm{a} & 11,33 \pm 4,49 \mathrm{a} & 11,01 \pm 4,67 \mathrm{a}\end{array}$

Médias na mesma coluna seguidas de letras distintas diferem ao nível de 5\% de significância pelo teste ANOVA one-way.

A análise do comportamento dos pais revelou que as fêmeas passam mais tempo no ninho que os machos. Em relação a alimentação, foram observados três comportamentos distintos: se alimentar, alimentar o parceiro ou os filhotes e beber água. Os machos se alimentaram mais frequentemente, porém eles também alimentaram as fêmeas através da regurgitação direto no bico. As fêmeas assim que se alimentavam voltavam para dentro do ninho para alimentar os filhotes. Os machos tiveram um maior comportamento de "grooming", que é a limpeza das penas com o bico, e este comportamento era realizado sempre no poleiro superior e em frente ao ninho.
Em relação aos locais de preferência dentro da gaiola, o poleiro superior é o preferido por ambos, seguido do ninho para a fêmeas, e do poleiro inferior e comedouro de sementes para ambos (Tabela 10). O comedouro de sementes ficava localizado no fundo do gaiola. Durante a alimentação, as aves simulavam o comportamento de forragear para obter o alimento (Figura 2).

Também foram observados comportamentos de interação social positiva, em que os machos realizavam o "grooming" nas fêmeas mais frequentemente do que as fêmeas nos machos. Os filhotes foram observados saindo do ninho pela primeira vez a partir do $16^{\circ}$ dia de experimento já com o empenamento completo.

Tabela 10 - Médias de frequência de visitas aos locais dentro da gaiola pelas aves das diferentes fases, seguida pelo desvio padrão correspondente

\begin{tabular}{lccc}
\hline Locais & Suplemento alimentar & Homeopatia & Controle \\
\hline GN & $1,50 \pm 0,71 \mathrm{~b}$ & $1,52 \pm 0,43 \mathrm{a}$ & $1,17 \pm 0,31 \mathrm{a}$ \\
PS & $3,45 \pm 0,67 \mathrm{a}$ & $3,24 \pm 0,48 \mathrm{~b}$ & $3,32 \pm 0,65 \mathrm{~b}$ \\
PI & $1,54 \pm 0,55 \mathrm{a}$ & $1,83 \pm 0,52 \mathrm{a}$ & $1,26 \pm 0,43 \mathrm{a}$ \\
N & $2,97 \pm 2,32 \mathrm{a}$ & $2,82 \pm 2,10 \mathrm{a}$ & $2,85 \pm 2,63 \mathrm{a}$ \\
CF & $1,12 \pm 0,47 \mathrm{a}$ & $1,04 \pm 0,25 \mathrm{a}$ & $1,17 \pm 0,50 \mathrm{a}$ \\
CS & $1,92 \pm 0,62 \mathrm{a}$ & $1,89 \pm 0,83 \mathrm{~b}$ & $1,76 \pm 0,63 \mathrm{~b}$ \\
B & $0,80 \pm 0,25 \mathrm{a}$ & $0,93 \pm 0,22 \mathrm{a}$ & $0,84 \pm 0,24 \mathrm{a}$ \\
\hline
\end{tabular}

Médias na mesma linha seguidas de letras distintas diferem o nível de $5 \%$ de significância pelo teste ANOVA one-way. Legenda: GN = grade do ninho; $\mathrm{PS}=$ poleiro superior; $\mathrm{PI}=$ poleiro inferior; $\mathrm{N}=$ ninho; $\mathrm{CF}=$ comedouro de farinhada; $\mathrm{CS}=$ comedouro de sementes; $\mathrm{B}$ $=$ bebedouro. 


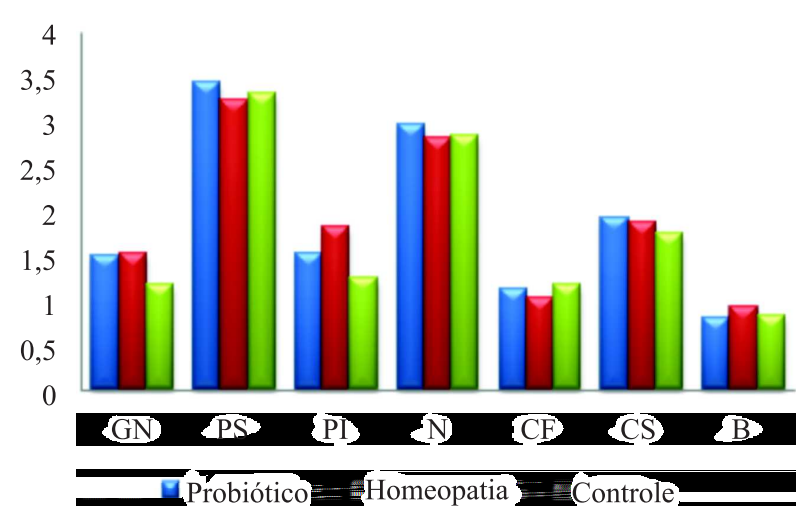

Figura 2 - Médias de frequência de visitas aos locais dentro da gaiola pelas aves das diferentes fases. Legenda: $\mathrm{GN}=$ grade do ninho; PS = poleiro superior; PI = poleiro inferior; $\mathrm{N}=$ ninho; $\mathrm{CF}$ $=$ comedouro de farinhada; $\mathrm{CS}=$ comedouro de sementes; $\mathrm{B}$ = bebedouro.

\section{DISCUSSÃO}

Segundo a análise do comportamento dos pais, os filhotes eram alimentados principalmente pela mãe, o que corrobora com o comportamento encontrado por Locatelli et al. (2013). Filho (1999) relatou que animais jovens recebem a microbiota principalmente das mães e que a transferência de microrganismos é muito eficiente em filhotes que são criados junto com os adultos.

Segundo Locatelli et al. (2013), o comportamento de "grooming" realizado sempre no poleiro superior e em frente ao ninho sugere-se que é para estimular o filhote a sair do ninho e realizar o mesmo comportamento. O comportamento de forrageio dos pais no fundo da gaiola é semelhante ao descrito por Sick (1997) como comportamento natural da espécie em vida livre, que tem o hábito de consumir as sementes que caem das plantas.

Os animais que receberam o suplemento alimentar demonstraram o maior ganho de peso entre os tratamentos. Tortuero (1973) indicou que filhotes de galinha que recebem probióticos durante o seu desenvolvimento apresentam melhoria dos índices zootécnicos, como aumento do ganho de peso e melhoria da conversão alimentar. Os animais tratados com homeopatia apresentaram um ganho de peso semelhante, porém um pouco menor em relação ao grupo que recebeu probiótico. Chabel (2009), em um estudo com ovinos tratados com uma combinação de Calcareas, obteve um resultado de ganho de peso de $12,1 \%$ superior ao grupo controle.
O consumo de farinhada foi semelhante nos três tratamentos, porém o consumo de sementes foi maior. Freitas (2016) observou, em um estudo de comportamento alimentar de calopsitas, que a preferência alimentar dos psitacídeos é a alimentação a base de sementes, quando ofertado sementes juntamente com farinhada.

\section{CONCLUSÃO}

Sendo assim, o uso da homeopatia é indicado pelo seu baixo custo, facilidade de administração, que pode ser na água ou na ração, sem necessidade de causar estresse ao animal, além de seu uso seguro, sem efeitos deletérios e sem deixar nenhum tipo de resíduo químico ou tóxico no ambiente ou no animal.

\section{LITERATURA CITADA}

ALTMANN, J. Observational study of behavior: sampling methods. Behaviour, v.40, p.227-267, 1974.

AOAC. 1995. Official Methods of Analysis of AOAC International. P. Cunniff (ed.). $16^{\text {th }}$ ed. Arlington. vol. I e II. $469 \mathrm{p}$.

BRIONES, S.F. Estudos sobre la aplicación de la homeopatia en producion animal. Santiago, Chile: [s.n], 1987. 45p.

BRUE, R. N. Nutrition. In: Harrison, G.; Lightfoot, T. Clinical Avian Medicine, 1994.

CHABEL, J.C.; VAN ONSELEN, V.J.; MORAIS, M.G.; NETO, I.M.C.; TEDESCHI, B.P. Efeito de um complexo homeopático "Homeobase Convert $\mathrm{H} 囚$ " em ovinos sob condições de restrição alimentar. Braz. J. vet. Res. anim. Sci., São Paulo, v.46, n.5, p.412-423, 2009.

COLLAR, N.J. Family psittacidae (parrots). In: DEL HOYO, J.; ELLIOTT, A.; SARGATAL, J. Handbook of the birds of the world. Barcelona: Lynx, 1997. p.280-479.

COSTA, N.C.C.; ARAÚJO, R.L.; FREITAS, G.B.L. Homeopatia: Um campo terapêutico fundamental no cuidado veterinário de animais de produção. Revista SalusGuarapuava (PR), v.3, n.2, p.75-89, 2009.

DIAS, A.F. Fundamentos da homeopatia - Princípios da prática homeopática. Ed. Cultura Médica. 2003.920p.

ECCH. The homeopathic treatment of animals in Europe. 2. ed. Nortfolk, UK: ECCH, 2003.

FARMACOPÉIA HOMEOPÁTICA BRASILEIRA. $2^{\mathrm{a}}$ ed. São Paulo: Editora Ateneu, 1997. 
FAUST, E.C.; D’ANTONI, J.S.; ODOM, V.; MILLER, M.J.; PERES, C.; SAWITS, W.; THOMEN, L.F.; TOBIE, J.; WALKER, J.H. A critical study of clinical laboratory technics for the diagnosis of protozoan cysts and helminth eggs in feces. Amer. J. Trop. Med., v.18, p.169-83, 1938.

FILHO, R.L.A.; SAMPAIO, H.M. Probióticos e prebióticos: realidade na avicultura industrial moderna. Education Journal CRMV·SP, São Paulo, v.2, n.3, p.59-71, 1999.

FORSHAW, J.M.; COOPER, W.T. Parrots of the world. Ed. Lansdowne, 1973. p.264-265.

FRANCISCO, L.R.; MOREIRA, N. Manejo, reprodução e conservação de psitacídeos brasileiros. Revista Brasileira de Reprodução Animal, v.36, n.4, p.215-219, 2012.

FREITAS, P.P. Comportamento alimentar de Nymphicus Hollandicus em ambiente artificial. Monografia de conclusão de curso. Brasília, 2016. 33p.

GRESPAN, A.; RASO, T. Psittacídeos. In: CUBAS, Z.S.; SILVA, J.C.R.; CATÃO-DIAS, J.L. (Org.). Tratado de animais selvagens. 2a ed. São Paulo-SP: Roca, v.1, p.613619, 2014.

HAHNEMANN, S. Organon da arte de curar. 6. ed. São Paulo: Robe Editorial, 1996.

HARCOURT-BROWN, N.H. Psittacine birds. In: TULLY, T.N.; LAWTON, M.P.C.; DORRESTEIN, G.M. Avian medicine. Elsevier, 2a ed., 2009. p.114-143.

JUNIPER, T.; PARR, M. Parrots: A guide to parrots of the world. New Haven: Yale Univ. Press, 1998. 584p.

IBAMA. PORTARIA no 93/1998, de 07 jul 1998 \& Alterações Importação e Exportação de Fauna Silvestre Nativa ou Exótica; Lista de Fauna Doméstica para fins de Operacionalização do Ibama. Pág. 1 de 8.

LOCATELLI, A.C.; WRUBLACK, S.C.; BASILE, L.F.; NASCIMENTO, A.F.; BERBER, G.C.M.; BERBER,
R.C.A. Comportamento reprodutivo e materno de araras Canindé (Ara ararauna) mantidas em cativeiro para conservação. Comunicata Scientiae, v.4, n.4, p.316-323, 2013.

MORENO, J.A. Homeopatia fácil. Ed. EHH. 2008. 206p.

NETO, R.M.B. Bases da homeopatia. Campinas: UNICAMP, 2006.

PAIVA, L.H.C. Os quatros pilares da homeopatia. Curso de formação de especialistas em homeopatia para farmacêuticos. Instituto Hanamanniano do Brasil. Rio de Janeiro, 1998. 17p.

RIBEIRO-FILHO, A. A institucionalização da homeopatia no Brasil. Revista de Homeopatia, n.71, p.70-73, jan./abr. 2008.

SICK, H. Ornitologia brasileira. Rio de Janeiro: Nova Fronteira, 1997.

SILVA, D.J. Análise de alimentos: métodos químicos e biológicos. 2a ed. Viçosa, MG: UFV, 1990. 165p.

SOARES FILHO, C.V.; CAETANO, H. Desempenho de bovinos de corte suplementados com Cr-levedura e produto homeopático. Araçatuba: UNESP, 2000. 21p.

STORACE, J.E.O. Reflexões sobre a homeopatia, história e epistemologia. Dissertação de mestrado. FACIS / IBEHE, Edição do autor, São Paulo, 2002.

TORTUERO, F. Influence of implantation of Lactobacillus acidophilus in chicks on the growth, feed conversion, malabsorption of fats syndrome and intestinal flora. Poultry Science, v.52, p.197-203, 1973.

VOCKEROTH, W. G. Veterinary Homeopathy: An overview. Can. Vet. J., v.40, p.592-594, 1999.

WYNDHAM, E. Environment and food of the budgerigar. Austi. J. Ecol., v.5, p.47-61, 1980.

Recebido para publicação em 26/02/2019 e aprovado em 27/12/2019. 